Ecclog:. 67(1), 1986, pp. 255-258

g 1986 by the Ecological Society of America

\section{THE IMPORTANCE OF STATISTICAL POWER WHEN TESTING FOR INDEPENDENCE IN ANIMAL MOVEMENTS ${ }^{1}$}

\section{Robert K. Swihart ${ }^{2}$ and Norman A. Slade ${ }^{2}$}

The home range (sensu Burt 1943) provides insight into facets of a species' social organization and foraging ecology (Metzgar 1979, Mitani and Rodman 1979, Madison 1980, Damuth 1981, Getty 1981, Mares et al. 1982, Hixon et al. 1983). Considerable effort has been expended deriving models of home range size (e.g., Calhoun and Casby 1958, Jennrich and Turner 1969, Koeppl et al. 1975, 1977, Schoener 1981, Anderson 1982, Don and Rennolls 1983). Reliability of home range estimates depends, to varying degrees, on the extent to which assumptions underlying these estimates are valid. For instance, statistical models of home range assume that locational observations are independent of one another (Dunn and Gipson 1977, Anderson 1982, Slade and Swihart 1983), i.e., that an animal's position at time $i$ is not a function of its position at time $i-\delta$. If successive observations are closely spaced in time this assumption probably is not valid, and home range size may be seriously underestimated (Swihart and Slade 1985a, b).

Schoener (1981) developed a statistic for detecting departures from independence of locational observations: $t^{2} / r^{2}$, the ratio of the mean squared distance between successive observations $\left(t^{2}\right)$ and the mean squared distance from the center of activity $\left(r^{2}\right)$. We empirically derived the sampling distribution of Schoener's $t^{2} / r^{2}$ ratio and provided a method for testing the null hypothesis of independence between successive observations (Swihart and Slade 1985a). For observations evenly spaced in time, we also suggested a procedure for determining the minimum time interval at which successive observations cease to be significantly correlated. A quasi-independent subset of points separated by this time interval can then be selected for use in analyses.

Recently Toft and Shea (1983) emphasized the utility of ecologists considering the relative costs of type I and type II errors for the statistical tests they use. In testing for independence, too low an $\alpha$ value may result in a biased home range estimate, whereas too high an $\alpha$ value may result in either too many useable data points being eliminated or data being gathered too infrequently. Typically, biologists rely on $\alpha$ levels of .05 or lower because $\alpha$ represents the probability of falsely rejecting $\mathrm{H}_{0}$, the null hypothesis (i.e., type I error; Sokal and Rohlf 1981), and the biologist's hypothesis is usually expressed as the alternative hypothesis $\left(\mathrm{H}_{1}\right)$. However, in selecting a time interval which will yield quasiindependent locational observations, we ultimately wish to accept the null hypothesis of independence, so we should actually be more concerned with the probability of falsely accepting $\mathrm{H}_{0}$ (i.e., $\beta$ or type II error). Because of this, we arbitrarily used an $\alpha$ of .25 with the hope that $\beta$ would be lowered to a "reasonable" level (Swihart and Slade 1985a). In this paper we evaluate our choice of $\alpha$ by generating power curves for our test of Schoener's ratio.

\section{Methods}

The quantity $1-\beta$, or the probability of rejecting a false $\mathrm{H}_{0}$, is the power of a test. Power should increase 


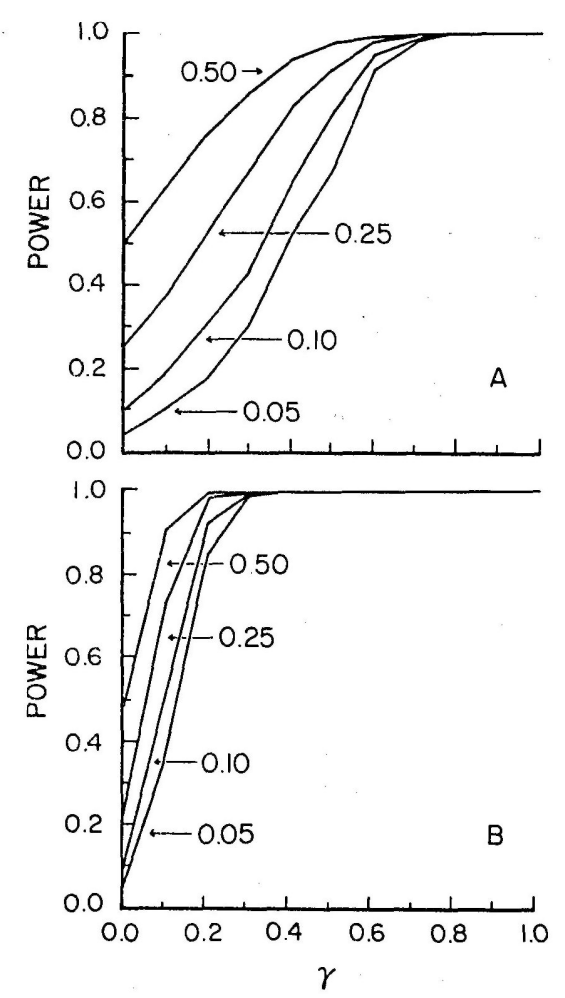

Fig. 1. Selected power curves for the test of independence of locational observations. $\gamma$ is a scalar measure of bivariate autocorrelation, defined in Methods. Only power curves constructed from sample sizes of $10(\mathrm{~A})$ and 100 (B) observations are illustrated; intermediate sample sizes $(n=30,50)$ produced power curves with intermediate characteristics relative to the curves depicted here. For each sample size, four levels of $\alpha$ were used: .05, .10,.25, and .50. Uniform (shown here) and normal error terms produced nearly identical power curves.

as truth (the actual $t^{2} / r^{2}$ ratio in our test) departs from the null hypothesis. The utility of power curves is that they enable comparisons of the effects that various $\alpha$ levels have on $\beta$ over a wide range of autocorrelations.

In general, first-order bivariate autocorrelation is described using a $2 \times 2$ matrix of autocorrelations and cross correlations (see Swihart and Slade 1985b). However, power curves typically are presented as plots of $1-\beta$ vs. a scalar analog of the statistic in question $\left(t^{2} / r^{2}\right.$ in this instance). Because we were unable to find any scalar measures of bivariate autocorrelation in the literature, we defined such a measure:

$$
\gamma=\left(\rho_{X_{i} X_{i-1}}+\rho_{Y_{i} Y_{i-1}}\right) / 2
$$

$\rho_{X_{i} X_{i-1}}$ and $\rho_{Y_{i} Y_{i-1}}$ are autocorrelations between $X$ at times $i$ and $i-1$ and $Y$ at times $i$ and $i-1$, respectively. No cross correlation terms were included in $\gamma$ because Schoener's ratio does not involve cross products. In our simulations we restricted our generation of values to cases in which the autocorrelation in each dimension was identical, i.e., $\rho_{X_{i} X_{i-1}}=\rho_{Y_{i} Y_{i-1}}=\gamma$, but with actual data $\rho_{X_{i} X_{i-1}}$ need not equal $\rho_{Y_{i} Y_{i-1}}$. Although $\gamma$ may assume values from -1 to 1 , movement paths characterized by negative autocorrelations, i.e., abrupt shifts from one side of the home range to the other, are difficult to envision. Hence, we focused on values of ? from 0 to 1 .

To construct power curves, a set of autocorrelated observations were required. If $X$ and $Y$ are independently distributed, autocorrelations of strength $\gamma$ may be generated using the equations

$$
X_{i}=\rho_{X_{i} X_{i-1}} \times X_{i-1}+\epsilon_{X}
$$

and

$$
Y_{i}=\rho_{Y_{i} Y_{i-1}} \times Y_{i-1}+\epsilon_{Y},
$$

where $\epsilon_{X}$ and $\epsilon_{Y}$ are random error terms for $X$ and $Y$, respectively. Using these equations we generated 1000 sets of locational observations of size $n$ for a variety of $\gamma$ values. For each $\gamma$, Schoener's ratio was calculated for each set, and a tally was made of the number of sets for which the null hypothesis of independence was rejected. Power curves were constructed for four levels of $\alpha(.05, .10, .25, .50)$ at each of four sample sizes $(n=$ $10,30,50,100)$ and two distributions of error terms (a bivariate uniform distribution over the unit square and a bivariate normal distribution with zero mean and unit variance).

Sample locations of an organism within its home range may not reflect the true shape of the home range. Because home range "shape" (i.e., eccentricity; Swihart and Slade 1985a) is important in calculating critical values of Schoener's ratio, we also constructed power curves based on critical values calculated using sample eccentricities rather than the parametric eccentricity of one. This served as a check on the power of the test as used by biologists under ordinary field conditions.

\section{Results and Discussion}

Power curves for Schoener's ratio are presented in Fig. 1. For all curves the power of the test increased (or, equivalently, the probability of a type II error decreased) as $\gamma$ increased. Increases in power were most pronounced for large sample sizes and high values of $\alpha$ (Fig. 1). For example, at $\gamma=0.1$ the probability of rejecting $\mathrm{H}_{0}$ was 0.635 for $n=10$ and $\alpha=.50$ (Fig. 1A), whereas the power of the test for the same $\alpha$ level was 0.906 when $n=100$ (Fig. 1B). Similarly, the power of the test at $\gamma=0.1$ and a sample size of 100 was 0.353 for $\alpha=.05$ and 0.906 for $\alpha=.50$ (Fig. 1B).

Using the observed estimate of eccentricity to calculate the critical value (Swihart and Slade 1985a) had little effect on the power of the test when sample sizes were large, but power declined slightly at small sample sizes. At least two factors contributed to underesti- 
mation of power at small $n$. First, small $n$ resulted in larger errors when estimating eccentricity, which in turn produced larger errors in the calculation of critical values for $t^{2} / r^{2}$. In fact, the standard deviation of sample eccentricities was nearly six times larger for $n=10$ compared to $n=100$ when $0<\gamma<0.2$. Second, $n=$ 10 was the lower limit at which the distributional assumptions necessary to calculate critical values of $t^{2} / r^{2}$ applied (Swihart and Slade 1985a). Nonetheless, the effects of sampling variation on the power of the test of independence appeared to be small. Thus, tests of locational data collected in the field should closely adhere to the power curves shown here.

Any choice of an $\alpha$ level must be tempered by the realization that $\beta$ will be affected as well (Toft and Shea 1983). When testing the null hypothesis of independence, the level of $\beta$ is at least as important as the level of $\alpha$; in general, then, power curves characterized by large positive slopes at low levels of $\gamma$ are preferable. High power at low levels of $\alpha$ indicates a small probability of type II error. Curves constructed for high (.50) and moderate (.25) $\alpha$ levels exhibited steep slopes associated with low values of $\gamma$ (Fig. 1).

We recommend testing for independence with an $\alpha$ level on the order of .25 or .50. Levels of $\alpha$ less than .25 seldom produced powers in excess of 0.50 unless $\gamma$ was greater than 0.25 (Fig. 1); thus, the probability of falsely accepting the hypothesis of independence is greater than 0.50 for low $\gamma$. Clearly, this is an unacceptably high value of $\beta$.

Using an $\alpha$ level of .50 was the most conservative and most powerful approach we tried; that is, $\beta$ was always smallest at this level of significance (Fig. 1). However, use of such a large $\alpha$ is twice as likely to lead to overestimation of the time interval necessary to achieve independence between successive observations, as compared to $\alpha=.25$. As a result, valuable data may be discarded prior to estimation of home range size. Or, if a pilot study is conducted to determine an appropriate sampling interval and $\alpha=.50$ is used, the estimated sampling interval may be longer than necessary to ensure collection of independent observations. Based on our admittedly limited experience with this test of independence, we have noticed that sampling intervals derived for small mammals $(<200$ g) using $\alpha$ levels ranging from .25 to .50 usually differ by no more than 20-30 min. However, we suspect that the magnitude of this difference may increase as a function of body mass in terrestrial mammals (see Lindstedt and Calder 1981, Calder 1983, 1984).

\section{Acknowledgments: $\quad$ D. John Anderson, Bradley J.} Bergstrom, and an anonymous reviewer provided constructive comments on the manuscript. This study was supported by an Honors Fellowship from the Univer- sity of Kansas Graduate School to R. K. Swihart and by University of Kansas General Research Grant 3509 0038 to N. A. Slade. Computing resources were proprovided by the University of Kansas Academic Computing Center.

\section{Literature Cited}

Anderson, D. J. 1982. The home range: a new nonparametric estimation technique. Ecology 63:103-112.

Burt, W. H. 1943. Territoriality and home range concepts as applied to mammals. Journal of Mammalogy 24:356362.

Calder, W. A., III. 1983. Ecological scaling: mammals and birds. Annual Review of Ecology and Systematics 14:213230.

230. 1984. Size, function, and life history. Harvard University Press, Cambridge, Massachusetts, USA.

Calhoun, J. B., and J. U. Casby. 1958. Calculation of home range and density of small mammals. United States Public Health Monograph 55:1-24.

Damuth, J. 1981. Home range, home range overlap, and species energy use among herbivorous mammals. Biological Journal of the Linnean Society 15:185-193.

Don, B. A. C., and K. Rennolls. 1983. A home range model incorporating biological attraction points. Journal of Animal Ecology 52:69-81.

Dunn, J. E., and P. S. Gipson. 1977. Analysis of radio telemetry data in studies of home range. Biometrics 33:85101.

Getty, T. 1981. Structure and dynamics of chipmunk home range. Journal of Mammalogy 62:726-737.

Hixon, M. A., F. L. Carpenter, and D. C. Patton. 1983. Territory area, flower density, and time budgeting in hummingbirds: an experimental and theoretical analysis. American Naturalist 122:366-391.

Jennrich, R. I., and F. B. Turner. 1969. Measurement of non-circular home range. Journal of Theoretical Biology 22:227-237.

Koeppl, J. W., N. A. Slade, K. Harris, and R. S. Hoffmann. 1977. A three-dimensional home range model. Journal of Mammalogy 58:213-220.

Koeppl, J. W., N. A. Slade, and R. S. Hoffmann. 1975. A bivariate home range model with possible applications to ethological data analysis. Journal of Mammalogy 56:8190.

Lindstedt, S. L., and W. A. Calder III. 1981. Body size, physiological time, and longevity of homeothermic animals. Quarterly Review of Biology 56:1-16.

Madison, D. M. 1980. Space use and social structure in meadow voles, Microtus pennsylvanicus. Behavioral Ecology and Sociobiology 7:65-71.

Mares, M. A., T. E. Lacher, Jr., M. R. Willig, N. A. Bitar, R. Adams, A. Klinger, and D. Tazik. 1982. An experimental analysis of social spacing in Tamias striatus. Ecology 63: 267-273.

Metzgar, L. H. 1979. Dispersion patterns in a Peromyscus population. Journal of Mammalogy 60:129-145.

Mitani, J. C., and P. S. Rodman. 1979. Territoriality: the relation of ranging pattern and home range size to defendability, with an analysis of territoriality among primate species. Behavioral Ecology and Sociobiology 5:241-251.

Schoener, T. W. 1981. An empirically based estimate of home range. Theoretical Population Biology 20:281-325.

Slade, N. A., and R. K. Swihart. 1983. Home range indices for the hispid cotton rat (Sigmodon hispidus) in northeastern Kansas. Journal of Mammalogy 64:580-590. 
Sokal, R. R., and F. J. Rohlf. 1981. Biometry. Second edition. W. H. Freeman, San Francisco, California, USA.

Swihart, R. K., and N. A. Slade. 1985a. Testing for independence of observations in animal movements. Ecology 66:1176-1184.

Swihart, R. K., and N. A. Slade. 1985b. Influence of sampling interval on estimates of home range size. Journal of Wildlife Management 49:1019-1025.

Toft, C. A., and P. J. Shea. 1983. Detecting community- wide patterns: estimating power strengthens statistical inference. American Naturalist 122:618-625.

${ }^{1}$ Manuscript received 30 November 1984; accepted 28 March 1985;

final version received 24 May 1985.

${ }^{2}$ Museum of Natural History

and Department of Systematics and Ecology; University of Kansas, Lawrence, Kansas 66045 USA. 